Aims Explore whether clinicians working in EDs address paediatric obesity, identify obstacles, and seek opinions on whether this can be improved.

Methods A survey was distributed to clinical staff seeing children in EDs through the PERUKI network. Data were analysed to identify current attitudes towards addressing obesity, obstacles, and ideas for improvement.

Results 693 responses were received from 57 sites. Current rates of addressing obesity are low. 127 (18.3\%) respondents address it with nearly every/every patient. Paediatric/ Paediatric Emergency Medicine (PEM) doctors are more likely than Non-paediatric/PEM doctors or Advanced Care/ Emergency Nurse Practitioners. (AC/ENP) (25.6\% v 12.3\%, $\left.\chi^{2}=20.26, \mathrm{p}<0.0001\right)$. Barriers included: lack of referral options $(78.6 \%)$, time $(77.8 \%)$, concern regarding negative responses (77.3\%), obesity being a familial issue (61\%) and lack of training (53.1\%). 'Concern regarding negative responses' was the most commonly cited barrier for those from Non-Paediatric/PEM doctors (83.2\%) and AC/ENP's (84.1\%). 'Lack of training' was higher amongst Non-Paediatric/PEM doctors (63.9\%) and AC/ENP's (71\%). 'Concern regarding negative responses' was also highest in those working $0-5$ yrs in ED (81.6\%), whereas 'Lack of time' was the biggest barrier in those working over 10 years in ED. To improve addressing obesity within EDs clinicians requested support with diagnosis, easier referral pathways, training, and changes in ethos both within departments and at local and national levels.

Conclusions Like other healthcare professionals, ED clinicians currently face many barriers in addressing obesity with their patients. However, by addressing these at a local and national level, the majority of ED clinicians feel they can have a role in helping to address the paediatric obesity crisis.

\section{G211 DESIGNING A CRITICAL CARE OUTREACH SERVICE - AN AUDIT AND NATIONAL SURVEY}

N Peshimam, S Stockinger, J Weber, R Mitting. Paediatric Intensive Care Unit, Imperial College Healthcare NHS Trust, London, UK

\subsection{6/archdischild-2020-rcpch.179}

Critical Care outreach services for adults have been shown to reduce mortality. It has also been demonstrated consistently that review of step-down patients on the ward reduces readmission to ICU and is therefore cost effective. There is, however, no published evidence on the benefit or lack thereof of critical care outreach services for children.

The Bedside PEWS score has been found to predict critical deterioration with a median score of 8 in deteriorating patients on paediatric wards.

A review of recent serious incident investigations within our NHS Trust identified a common theme of 'failure to escalate care in the deteriorating patient.'

Aims With a view to designing a critical care outreach programme, we completed a national survey of all British paediatric intensive care units to discover what percentage of units have a funded service, and whether this is staffed by nurses or doctors. We then carried out a review of the last 1 year of 'internal collapse' admissions from the paediatric wards to PICU within 1 NHS Trust to assess the time of day that critical care admissions most commonly occurred, and to confirm that a BPEWS of 8 would predict deterioration in our population.

Method A telephone survey of all PICUs listed in the PICANET database. A case note review of the previous 1 year of admissions to PICU from wards within the same hospital. Recorded was time of admission, BPEWS score at admission, and maximum BPEWS in the 12 hours prior to admission.

Results Of the 27 PICUs listed in the database, 9 have funding for a critical care outreach service. In all apart from 1 this was a nurse led service.

The mean and median PEWS scores for the internal collapse patients were 8 at the time of admission, and a mean maximum of 9 during the previous 12 hours.

$39 \%$ of admissions to PICU from paediatric wards occurred between 0800-1700 hrs, 45\% between 1400-2200 hrs and 26\% between 2200-0800 hrs.

Conclusion A third of PICUs had a dedicated critical care outreach service. A day-time only service would miss $25 \%$ of admissions. A BPEWS of less than 8 should be used as a trigger for review.

\section{G212 FEASIBLITY AND ACCEPTABILITY PILOT OF A PUBLIC HEALTH INTERVENTION DELIVERED IN THE PAEDIATRIC EMERGENCY DEPARTMENT}

${ }^{1,2}{ }^{R E}$ Isba, ${ }^{1}$ RL Edge. 'Lancaster Medical School, Lancaster University, Lancaster, UK; ${ }^{2}$ Emergency Department, North Manchester General Hospital, Manchester, UK

10.1136/archdischild-2020-rcpch.180

Aim Paediatric Emergency Departments (PEDs) are well-placed to deliver public health interventions. Whilst numerous studies describe the effectiveness of a range of ED-based interventions for adults, less has been done to assess interventions for Children and Young People (CYP).

Every year in England, millions of CYP attend hospital, often with relatively minor illnesses/injuries, which sometimes result in long waits - time that could be used to improve wider health and wellbeing.

This pilot study assessed the feasibility and acceptability of delivering a public health intervention in the PED of a busy district general hospital.

Methods Full prospective ethical approval was obtained. Participants were CYP and their carers attending a PED in England. An opportunistic sampling strategy was used, with a focus on recruiting those who had a wait whilst in the department.

The intervention was a consultation delivered by a public health specialist, based around the 'Screening, Brief Intervention, and Referral for Treatment' (SBIRT) model and focussed on: household smoking, vaccination status, dental health, and frequent attendance.

Quantitative outcome data (e.g. registering with dentist) were collected by phone at one week and then one, three, and six months post-enrolment (where indicated). Qualitative data came from engaging with participants and completion of a field diary by the public health specialist (primary researcher).

Results Thirty participants were recruited over the two-week pilot, with $50 \%$ of CYP participating in the consent process. Twenty participants (67\%) triggered at least one screening question, with dental health and (household) smoking being the most common triggers. 
Four participants were lost to phone follow-up at one week and a further five were 'thanked and discharged' as they had not triggered any of the categories during screening. Of the remaining participants, five had taken action as a result of the study and others had plans, all relating to dental appointments. Follow-up is ongoing and due for completion in March 2020.

Conclusion The PED offers an underutilised opportunity to deliver public health interventions. Findings from this study will be used to refine the intervention before an assessment of its effectiveness is made, using an appropriate study design.

Acknowledgements This study was funded by a grant from the Sir Halley Stewart Trust.

\section{G213 A NEW TECHNIQUE FOR ULTRASOUND-GUIDED CENTRAL VENOUS CATHETERIZATION IN PEDIATRICS (SYRINGE FREE APPROACH)}

${ }^{1,2} \mathrm{~A}$ Jorya, ${ }^{1,2,3} \mathrm{M}$ Naeem, ${ }^{1,2,3} \mathrm{M}$ Arabi, ${ }^{1,2,3} \mathrm{~A}$ Alshihri. ${ }^{1}$ Department of Pediatrics, Ministry of National Guard Health Affairs, Riyadh, Saudi Arabia; ${ }^{2}$ King Abdullah International Medical Research Center, Riyadh, Saudi Arabia; ${ }^{3}$ College of Medicine, King Saud bin Abdulziz University, Riyadh, Saudi Arabia

\subsection{6/archdischild-2020-rcpch.181}

Background Central line insertion, a very common and invaluable procedure in PICU has undergone a major improvement with the advent of ultrasound assistance. In the process of further refinement, literature shows a comparison of various techniques. For the aim of increasing the safety profile and decrease the time required for CVL insertion, a new technique has been introduced by the author. We tested this technique against the other techniques that have been used in tertiary PICU unit in KSA.

Methods We prospectively monitored all vascular access procedures guided by ultrasound from March 2018 to March 2019. All patients who underwent central line insertion were observed by an independent nurse recorder that was not involved in the procedure. The observer recorded the patient age, gender, weight and BMI, diagnosis, indication for insertion, blood pressure state, insertion time, size of the line, number of pricks and arterial punctures if happened

Results Central line was inserted in 141 out of 800 total admissions during the study period. The author applied Syringe-free technique in 16 patients while in 125 patients central line was inserted via transverse axis out of plane technique. For the syringe-free group: Mean age was 49 months. Mean weight was $13.6 \mathrm{~kg}$ and mean BMI WAS 15.2. The femoral vein was the selected site of insertion in 13 patients $81 \%$. The mean time of insertion was 86 seconds with a mean of attempts was 1.1. For the transverse technique: Mean age was 39 months. Mean weight was $13.9 \mathrm{~kg}$ and mean BMI WAS 15.3.

Femoral vein was the selected site of insertion in 74 patients (59\%). The mean time of insertion was 304 seconds with a mean number of attempts of 1.38 .

Conclusion Syringe free technique is a safe procedure that can decrease the time of insertion by $400 \%$ and allow a continuous real time-US monitoring of the CVL insertion procedure.

\section{G214 IMPLEMENTATION OF A CAMHS STICKER FOR IMPROVED DOCUMENTATION OF MENTAL HEALTH PATIENTS PRESENTING TO THE CHILDREN'S EMERGENCY DEPARTMENT}

B Cuellar, R Sunley. Paediatric Emergency Department, University Hospitals Bristol NHS Trust, Bristol, UK

\subsection{6/archdischild-2020-rcpch.182}

Aims To devise and implement a new documentation sticker to be used by the CAMHS team (child and adolescent mental health services) in the Paediatric Emergency Department medical notes. To clearly document safety plans, diagnosis and follow up to the hospital and community teams co-caring for mental health patients.

Method Twenty sets of notes were taken at random from the year 2017-2018. The documentation of information in the notes, written by the CAMHS team, and quality of the discharge letter, written by the Emergency Department medical team, were assessed. The CAMHS sticker was introduced for the CAMHS team to use to document their assessment. Twenty sets of notes were assessed after the implementation of the CAMHS sticker.

Results There was improved documentation after the implementation of the CAMHS sticker in the notes. Specifically, the CAMHS team documentation of their assessment (Pre 40\%: Post 85\%), management plan (Pre 70\%: Post 100\%) and risk assessment (Pre 30\%: Post 90\%). The quality of the emergency department discharge letter also improved for diagnosis (Pre 90\%: Post 95\%) and risk assessment (Pre 30\%: Post $70 \%$ ). However, there was a fall in documentation in the management plan in the discharge letter (Pre 90\%: Post 55\%) for reasons unknown.

Conclusion The introduction of the CAMHS sticker has improved documentation and communication between clinical teams and primary care. This can be seen in all areas in the notes and discharge letter. However, documentation of the management plan in the discharge letter needs to be refined. It was also noted that the name, contact number, date and time of assessment by the CAMHS team could be improved. A new sticker has been designed to include these demographics in order for clearer handover between the CAMHS team and the emergency department.

The introduction and the positive effects the CAMHS sticker has had on the working relationship between the CAMHS team and the emergency department has been noted by the inpatient teams. The CAMHS sticker has now been revised by the inpatient teams in order for them to use for the mental health patients on the wards. A re-audit of the use of the stickers should be done at a later date to ensure compliance is improving as well as documentation.

\section{G215 ECMO AND AUDIOLOGICAL FOLLOW UP IN CHILDREN}

${ }^{1} \mathrm{CS}$ Cockburn, ${ }^{2} \mathrm{M}$ Davidson, ${ }^{2} \mathrm{P}$ Donnelly, ${ }^{2} \mathrm{~N}$ Matta, ${ }^{2} \mathrm{G}$ Wylie, ${ }^{3} \mathrm{M}$ Law, ${ }^{3} \mathrm{Y}$ Sasaki. ${ }^{1}$ General Paediatrics, RHC Glasgow, Glasgow, UK; ${ }^{2} P I C U$, RHC Glasgow, Glasgow, UK; ${ }^{3}$ Medical Student, University of Glasgow, Glasgow, UK

\subsection{6/archdischild-2020-rcpch.183}

Introduction Children who have been supported with extracorporeal membrane oxygenation (ECMO) require follow-up including neurodevelopmental screening and audiological 